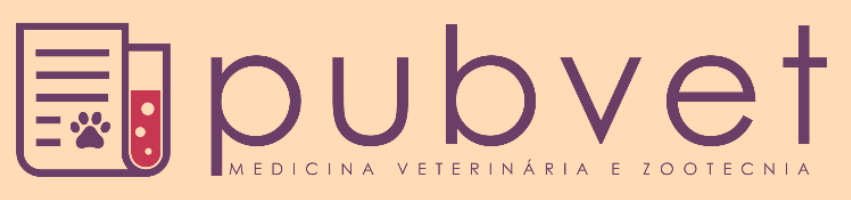

https://doi.org/10.31533/pubvet.v13n9a410.1-7

\title{
Princípios básicos na formulação de rações
}

\author{
Kátia Maria Cardinal ${ }^{1}$, João Luiz Benavides $\operatorname{Costa}^{2}{ }^{\circ}$, Andréa Machado Leal Ribeiro ${ }^{3} \bullet$ \\ ${ }^{I}$ Aluna de doutorado do programa de pós-graduação em Zootecnia, Universidade Federal do Rio Grande do Sul, Porto Alegre- RS. \\ ${ }^{2}$ Doutor em Produção Animal, Universidade Federal do Rio Grande do Sul, Porto Alegre- RS. \\ ${ }^{3}$ Professora do departamento de Zootecnia, Universidade Federal do Rio Grande do Sul, Porto Alegre - RS. \\ Autor para correspondência, E-mail:katia.zootecnia@hotmail.com
}

\begin{abstract}
Resumo. O desempenho dos animais de uma criação está intimamente relacionado com o programa nutricional desenvolvido na propriedade. As definições das características dos animais e dos alimentos que estarão envolvidos no programa nutricional são de fundamental importância, tornando possível formular uma ração que atenda as necessidades nutricionais dos animais e que otimize a utilização dos alimentos. Desta forma, o objetivo desta revisão é abordar os princípios básicos que devem ser considerados para a formulação de uma ração e apresentar, de forma didática, exemplos de formulação de ração que podem ser realizados manualmente ou com o auxílio de uma calculadora.
\end{abstract}

Palavras chave: Alimentação, dieta, exigências nutricionais

\section{Basic principles in diets formulation}

\begin{abstract}
The performance of farm animals is closely related to the nutritional program developed on the property. Making the definitions of the characteristics of the animals and food that will be involved in the nutritional program is of fundamental importance, as it will make it possible to formulate a ration that will meet the nutritional needs of the animals and that will optimize the utilization of the food. Thus, the aim of this review is to present the basic principles that must be considered for the formulation of a feed and to demonstrate, in a didactic way, examples of feed formulation that can be performed manually or with the aid of a calculator.
\end{abstract}

Key words: Food, diet, nutritional requirements

\section{Principios básicos en la formulación de raciones}

Resumen. El rendimiento de los animales de granja está estrechamente relacionado con el programa nutricional desarrollado en la propiedad. Las definiciones de las características de los animales y los alimentos que participarán del programa nutricional son de fundamental importancia, ya que permitirá formular una ración que satisfaga las necesidades nutricionales de los animales y que optimice la utilización de los alimentos. Por lo tanto, el propósito de esta revisión es presentar los principios básicos que deben considerarse para la formulación de un alimento y demostrar, de manera didáctica, ejemplos de formulación de alimentos que pueden realizarse manualmente o con la ayuda de una calculadora.

Palabras clave: Alimentación, dieta, requerimientos nutricionales

\section{Introdução}

A produção animal do Brasil está entre as mais desenvolvidas do mundo e o país é destaque como um dos maiores produtores e exportadores de proteína de origem animal (FAPRI, 2019). Entre 2017 e 2018, a agropecuária contribuiu com 21,6\% do PIB nacional, sendo que só a pecuária representou 6,6\% desse total e $31 \%$ do PIB do agronegócio (ANUALPEC, 2019). Para atingir esse status produtivo, houve investimentos e investigação científica na produção animal, sendo que a nutrição animal é destaque, pois representa 70 a $80 \%$ dos custos totais de produção de diversas espécies (Valadares Filho et al., 2016). 
A formulação de rações tem por objetivo traduzir as necessidades nutricionais teóricas em necessidades alimentares reais (Andriguetto et al., 2003), ou seja, é definir a quantidade de alimentos que devem ser fornecidos para um animal durante o período de 24 horas, sendo que o conjunto de alimentos deve estar equilibrado nutricionalmente; assim será ingerido em quantidades suficientes e assegurará o fornecimento dos nutrientes exigidos pelos animais para pleno desenvolvimento e manutenção das funções vitais. Segundo NRC (2000) a formulação da ração pode ser dividida em três etapas: estimativa das exigências nutricionais dos animais, cálculo dos nutrientes fornecidos pelos alimentos e modelagem do problema para a obtenção de uma ração que possa atender as exigências dos animais. Este processo é de fundamental importância na criação de animais domésticos e está intimamente relacionada com a viabilidade técnica e econômica do sistema de produção animal.

Existem vários softwares para formulação de ração e existem métodos manuais, como o método de tentativa, quadrado de Pearson e o método algébrico. Assim, essa revisão teve como objetivo sumarizar princípios básicos a serem observados para a formulação de ração e descrever dois métodos de formulação, algébrico e quadrado de Pearson, que podem ser realizados com o auxílio de uma calculadora.

\section{Caracterização dos animais e definição das exigências nutricionais}

Para a formulação de ração é necessário, primeiramente, caracterizar o animal para qual a ração será formulada e, posteriormente, analisar quais serão as exigências nutricionais que deverão ser atendidas em função da aptidão e da fase produtiva. Existem diversos fatores relacionados aos animais que devem ser considerados, como a espécie, idade, sexo, raça, tamanho e as condições ambientais. Os diferentes conjuntos de características resultarão em necessidades nutricionais distintas, que devem ser seguidas para que se obtenha um programa de nutrição eficiente (NRC, 2001; Rostagno et al., 2017). Desta forma, uma fêmea bovina produzindo leite tem exigência diferente de um macho bovino em fase de terminação. Em frangos de corte com crescimento acelerado, a exigência dos nutrientes muda rapidamente e, para atender essas alterações, são utilizadas de quatro a cinco fórmulas de ração durante os 42 dias produtivos. Como pode ser visto na tabela abaixo, as exigências de proteína e as de energia são alteradas conforme a idade do animal avança:

Tabela 1- Exigência de energia e proteína para frangos de corte machos de desempenho superior

\begin{tabular}{llcccc}
\hline & \multicolumn{5}{c}{ Idade, dias } \\
\cline { 3 - 6 } & & $1-7$ & $8-21$ & $22-33$ & $34-42$ \\
\hline Energia Metabolizável & $\mathrm{Kcal} / \mathrm{kg}$ & 2960 & 3050 & 3150 & 3200 \\
Proteína & $\%$ & 22,11 & 21,14 & 19,73 & 18,31 \\
\hline
\end{tabular}

Adaptada de Rostagno et al. (2017).

Para a definição das exigências nutricionais dos animais, o formulador deve consultar tabelas de exigências já publicadas, como as do National Research Council (NRC) para bovinos de corte, bovinos leiteiros, pequenos ruminantes, frangos e suínos (NRC, 1989, 1994, 2000, 2001, 2007). No Brasil existem as tabelas brasileiras para aves, suínos e bovinos, publicadas pela Universidade Federal de Viçosa (Rostagno et al., 2017; Valadares Filho et al., 2016) que são periodicamente atualizadas. Majoritariamente, as tabelas costumam ter como entrada as características do animal e como resposta a exigência de cada nutriente e de energia. É fortemente recomendado que o formulador não misture dados de diferentes tabelas; isto é, defina-se por uma tabela e somente em caso de falta de alguma informação, busque outra.

\section{Seleção dos alimentos}

Alimento é um material que, após ser ingerido pelo animal, tem a capacidade de ser digerido em seus nutrientes principais, que após serão absorvidos e utilizado para diversos fins. O termo alimento é utilizado de forma generalista, pois nem todos os constituintes de um alimento são passíveis de digestão. Desta forma, a parte que pode ser absorvida e utilizada pelos animais é chamada de nutriente (Berchielli et al., 2011). Para um alimento ser utilizado como ingrediente na formulação de uma ração, é necessário conhecer as suas propriedades físicas e químicas, pois desta forma é possível otimizar a eficiência de 
utilização dos alimentos pelos animais. Plantas e animais contêm tipos similares de nutrientes que podem ser agrupados conforme sua constituição, propriedades e funções (Berchielli et al., 2011; Prado, 2010). As principais partes componentes dos alimentos são:

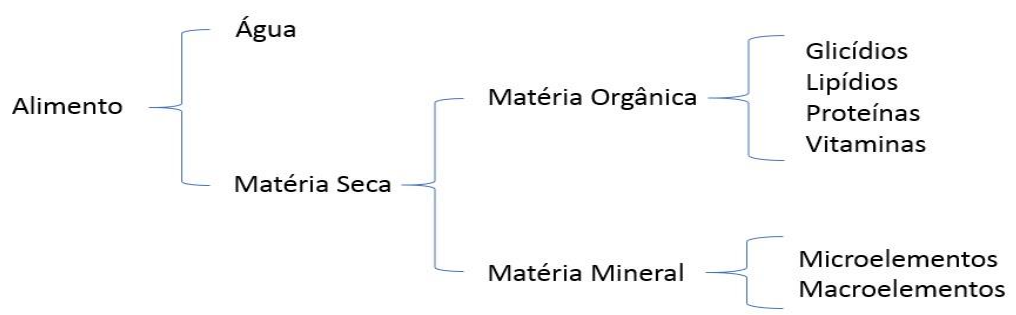

Figura 1. Composição química dos alimentos. Adaptado de Andriguetto et al. (2003).

Os alimentos também podem ser classificados como volumosos e concentrados. Os alimentos volumosos são aqueles que contêm alto teor de fibra bruta e baixo valor energético, com menos de $60 \%$ nutrientes digestíveis totais e mais de $18 \%$ de fibra bruta. Já os alimentos concentrados são aqueles que apresentam menos de $18 \%$ de fibra bruta em sua composição, porém contêm alto teor energético ou proteico. Alimentos concentrados energéticos apresentam menos de $20 \%$ de proteína bruta, e os concentrados proteicos apresentam mais de $20 \%$ de proteína bruta em sua composição (Giger-Reverdin et al., 2002; Ibarra et al., 2017).

A escolha dos alimentos dependerá do balanceamento de nutrientes desejado. Devem ser levadas em consideração as limitações existentes para o uso de alguns ingredientes, como problemas de toxidade, manuseio e conservação. E é necessário também obter informações sobre a disponibilidade dos alimentos na região e que o custo seja acessível (Lana, 2007; Rezende et al., 2011). As informações nutricionais dos alimentos e a porcentagem que é possível incluir na dieta podem ser encontradas nos NRCs (NRC, 1989, 1994, 2000, 2001, 2007, 2012) e nas tabelas brasileiras de aves e suínos e em trabalhos científicos, como pode ser visto na Tabela 2.

Tabela 2. Teores de matéria seca (MS, \%), nutrientes digestíveis totais (NDT, \% MS), proteína bruta (PB, \% MS) e fibra em detergente neutro (FDN, \% MS) em concentrados energéticos para vacas leiteiras

\begin{tabular}{llcccc}
\hline Alimentos & Tipo & MS & NDT & PB & FDN \\
\hline Concentrados energéticos & & & & \\
\hline Arroz & Farelo & 88,07 & 79,50 & 14,41 & 34,65 \\
Arroz & Farelo Desengordurado & 89,33 & - & 17,41 & 26,65 \\
Laranja & Polpa Seca & 87,51 & 78,00 & 7,06 & 25,15 \\
Laranja & Bagaço Silagem & 13,97 & 78,00 & 8,48 & 24,58 \\
Mandioca & Farinha de Varredura & 91,51 & 70,64 & 2,38 & 12,20 \\
Mandioca & Raiz dessecada & 43,85 & - & 2,40 & - \\
\hline
\end{tabular}

Adaptado de Cappelle et al. (2001) e Lana (2007).

Após a caracterização dos animais, definição das exigências nutricionais e da escolha dos ingredientes, é necessário optar pelo melhor método de formulação de ração, de acordo com o conhecimento, conveniência e precisão desejada.

\section{Formulação de rações}

Existem diversos métodos para formulação de rações e os mais práticos são aqueles realizados por softwares ou planilhas eletrônicas, pois efetuam a formulação com diversos ingredientes e nutrientes de forma rápida, precisa e visando o custo mínimo (NRC, 1989, 1994, 2000, 2001, 2007, 2012). O software Super CRAC $^{\circledR}$ foi o primeiro programa de formulação de ração de custo mínimo lançado no Brasil, em 1983, e nele podem ser formuladas rações, sais minerais e premix de custo mínimo para atender as exigências nutricionais de aves, suínos, bovinos, caprinos, ovinos, cães, gatos, peixes e coelhos. Outro exemplo de software é o Optimal WinDiet ${ }^{\circledR}$, que é fornecido em uma versão acessível para produtores e outra para prestadores de serviços, fábricas de ração etc. Para formulação de ração com softwares, 
alguns programas são mais complicados que outros e exigem conhecimento avançado sobre os alimentos e nutrição animal. No entanto, também existem métodos manuais simples que podem ser utilizados para obtenção das proporções dos alimentos, como os métodos de tentativa, algébrico ou equações simultâneas e quadrado de Pearson (Lana, 2007). A seguir serão exemplificados, de forma didática, os métodos algébricos e quadrado de Pearson.

\section{Método algébrico:}

O método consiste em estabelecer e resolver um sistema de equações, sendo as incógnitas os ingredientes a serem utilizados na ração. Este método permite a mistura de dois ou mais ingredientes e se torna mais complexo à medida que o número de ingredientes e nutrientes são maiores (Rostagno et al., 2017).

\section{Exemplo prático:}

Para uma vaca de raça leiteria de frame grande, com $455 \mathrm{~kg}$ de peso vivo, produzindo 20 litros de leite/dia no meio da lactação, com ganho de peso de $300 \mathrm{~g} / \mathrm{dia}$ e capaz de ingerir $16 \mathrm{~kg} \mathrm{MS} / \mathrm{dia}$ (CMS), a exigência proteica é de $15,2 \%$ ou 2,4 $\mathrm{kg} \mathrm{PB} /$ dia e a energética é de 23,2 Mcal/dia ou 1,45 Mcal/kg/dia (NRC, 2001). Será considerado que esse animal consume $9,6 \mathrm{~kg}$ matéria seca (MS) de silagem de milho, $80 \mathrm{~g}$ de sal branco e $20 \mathrm{~g}$ de premix mineral por dia, e que se deseja completar a dieta com farelo de soja e milho moído para atender as exigências de proteína bruta (Tabela 3).

Tabela 3. Valores de proteína bruta e energia liquida de produção referentes a exigência nutricional do animal e composição dos alimentos utilizados do exemplo prático

\begin{tabular}{lccccc}
\hline & $\begin{array}{c}\text { Consumo de matéria } \\
\text { seca, } \mathrm{kg}\end{array}$ & \multicolumn{2}{c}{ Proteína bruta } & \multicolumn{2}{c}{ Energia liquida de produção } \\
\cline { 3 - 6 } & 16 & $\%$ & $\mathrm{~kg}$ & $\mathrm{Mcal} / \mathrm{kg}$ & Mcal/dia \\
\hline Exigência do animal & & 15,2 & 2,43 & 1,45 & 23,2 \\
\hline Alimentos & 9,2 & 8,8 & 0,81 & 1,45 & 13,34 \\
\hline Silagem de milho & $\boldsymbol{X}$ & 9,4 & $*$ & 2,01 & $*$ \\
Milho moído & $\boldsymbol{Y}$ & 43 & $*$ & 2,72 & $*$ \\
Farelo soja & 0,8 & - & - & - & - \\
Sal branco & 0,2 & - & - & - & - \\
Premix mineral & 5,8 & - & 1,62 & - & 9,86 \\
Déficit ${ }^{1}$ & & & & & \\
\hline
\end{tabular}

${ }^{1}$ Valores referentes as exigências do animal subtraído do fornecido pela silagem de milho, sal branco e premix mineral.

Para que o problema seja resolvido, monta-se o um sistema de equações lineares segundo os seguintes passos:

1. São estabelecidas as variáveis utilizadas no cálculo:

$\mathrm{X}=$ Milho moído e $\mathrm{Y}=$ Farelo de soja

2. Monta-se o sistema de equações:

EQ1 - Kg Milho moído + kg Farelo de soja = Déficit de MS $(\mathrm{kg})$

$\mathrm{EQ} 1-X+Y=5,8$

EQ2 $-\%$ PB do Milho $\times$ kg de Milho $+\%$ PB da Soja $\times$ kg de Soja $=$ Déficit PB $(\mathrm{kg})$

$\mathrm{EQ} 2-0,094 X+0,43 Y=1,62$

3. Multiplicam-se os termos da EQ1 por -0,094 (\% PB do milho) para ser possível resolver o sistema ao isolar uma das variáveis pelo método de eliminação:

$$
\begin{aligned}
\mathrm{EQ} 1--0,094 X-0,094 Y & =-0,545 \\
\mathrm{EQ} 2-0,094 X+0,43 Y & =1,62 \\
0-0,336 Y & =1,075 \\
Y & =3,199
\end{aligned}
$$


4. $\mathrm{O}$ valor determinado de $\mathrm{Y}$ é inserido na $\mathrm{EQ1}$ ou $\mathrm{EQ} 2$ para determinar o valor de $\mathrm{X}$

$$
\begin{array}{ll}
\mathrm{EQ} 1-X+3,199=5,8 & \mathrm{EQ} 2-0,094 X+(0,43 \times 3,199)=1,62 \\
\mathrm{EQ} 1-X=5,8-3,199=2,6 & \mathrm{EQ} 2-X=0,244 / 0,094=2,6
\end{array}
$$

\begin{tabular}{|c|c|c|c|c|c|}
\hline & \multirow{2}{*}{$\begin{array}{c}\text { Consumo de matéria } \\
\text { seca, } \mathrm{kg}\end{array}$} & \multicolumn{2}{|c|}{ Proteína bruta } & \multicolumn{2}{|c|}{ Energia líquida de produção } \\
\hline & & $\%$ & $\mathrm{~kg}$ & $\mathrm{Mcal} / \mathrm{kg}$ & Mcal \\
\hline Silagem de milho & 9,2 & 8,8 & 0,81 & 1,45 & 13,34 \\
\hline Milho moído & 2,6 & 9,4 & 0,24 & 2,01 & 5,226 \\
\hline Farelo soja & 3,2 & 43 & 1,38 & 2,72 & 8,704 \\
\hline Sal branco & 0,8 & - & - & - & - \\
\hline Premix mineral & 0,2 & - & & - & - \\
\hline Total & 16 & - & 2,43 & - & 27,27 \\
\hline
\end{tabular}

As quantidades de milho moído e farelo de soja para atender as necessidades proteicas diárias da vaca em questão são de 2,6 e 3,2 kg, respectivamente (Tabela 4).

Tabela 4. Composição da dieta após determinação das quantidades de milho moído e farelo de soja - método algébrico.

\section{Quadrado de Pearson:}

O método consiste em calcular a ração levando em consideração o valor percentual de um determinado nutriente. $\mathrm{O}$ método estabelece as proporções entre dois alimentos ou de duas misturas de alimentos, e o valor obtido para a proteína será intermediário ao teor de proteína dos dois alimentos misturados. Adaptando as informações contidas em Sakomura \& Rostagno (2007), descrevemos os seguintes passos para a realização do Quadrado de Pearson: 1) Desenhar um quadrado e colocar a porcentagem desejada do nutriente no centro do quadro; 2 ) colocar o conteúdo do nutriente (\%) de cada alimento nos ângulos esquerdos do quadrado; 3) subtrair diagonalmente no quadrado os menores números dos maiores e colocar os resultados nos ângulos direitos do quadrado; e; 4) a leitura dos resultados deve ser feita na horizontal e não na diagonal; 5) as quantidades de cada alimento serão expressas em porcentagem do total. As exigências para este método são que: 1) a base de referência (matéria seca ou matéria natural) deve ser a mesma para a exigência e o teor de nutrientes nos alimentos; 2) 5) é necessário que o número do centro do quadrado esteja entre os valores dos números dos ângulos esquerdos.

\section{Exemplo prático:}

Utilizaremos os mesmos dados do exemplo anterior para facilitar a visualização das diferenças entre os dois métodos. Os passos para a resolução pelo método de Pearson são os seguintes:

1. Definir, a partir de uma regra de três simples, o valor de referência do interior do quadrado transformando a necessidade de PB de quilogramas para o percentual sobre o CMS a ser atendido.

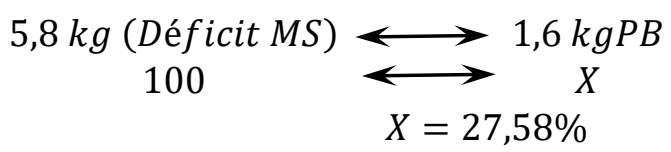

2. Posicionar o valor da porcentagem de $\mathrm{PB}$ dos ingredientes a serem utilizados nas extremidades do lado esquerdo do quadrado. Subtrair do valor de referência, no sentido diagonal, o valor de cada ingrediente para determinarmos a quantidade em partes de cada ingrediente.

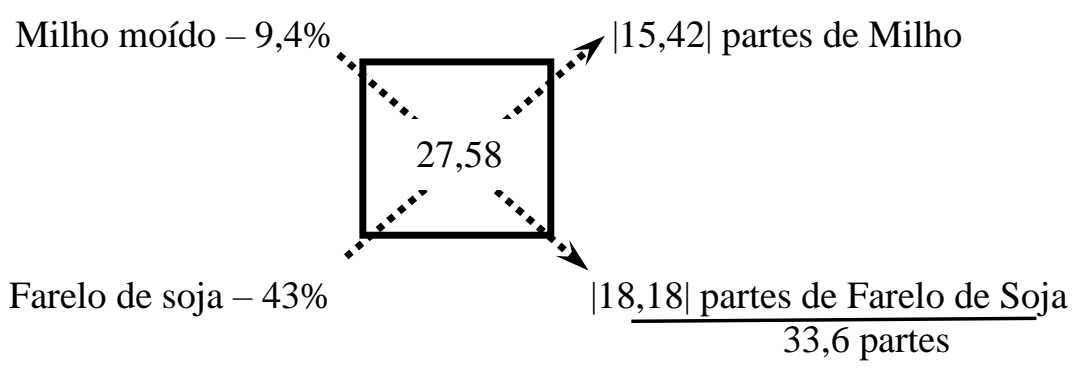


3. Com base na quantidade de partes de cada ingrediente, do total parte e do total de CMS disponível para ser utilizado com milho e soja deve-se fazer uma regra de três para determinar quanto de cada ingrediente deverá ser utilizado.

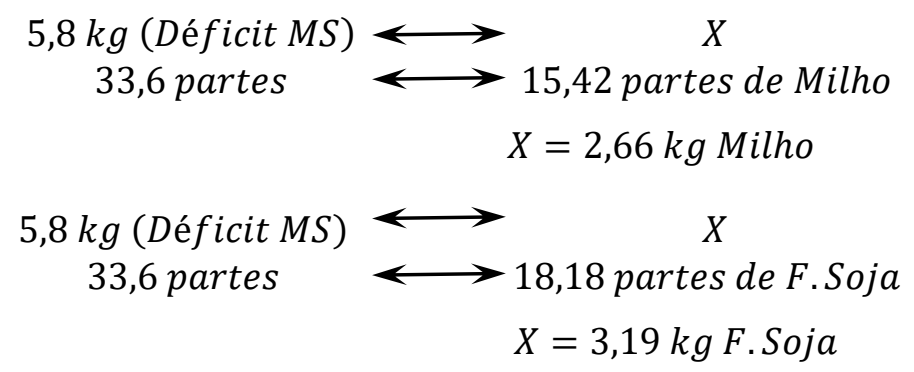

Obs.: Como alternativa, é possível realizar a primeira operação para determinar o valor de um dos ingredientes e, para determinar o valor do segundo ingrediente, subtrair o valor já determinado do valor total do déficit de matéria seca. Exemplo:

$$
\begin{aligned}
& 5,8 \mathrm{~kg} \text { (Déficit } M S) \longleftrightarrow \quad X \\
& 33,6 \text { partes } \longleftrightarrow 15,42 \text { partes de Milho } \\
& X=2,66 \mathrm{~kg} \text { Milho } \\
& \text { F. Soja }=5,8 \mathrm{~kg} \text { (Déficit MS) - 2,66 (Kg milho) }
\end{aligned}
$$

Farelo de soja $=3,19 \mathrm{~kg}$

Tabela 5. Composição da dieta após determinação das quantidades de milho moído e farelo de soja - método Quadrado de Pearson

\begin{tabular}{lccccc}
\hline & & \multicolumn{2}{c}{ Proteína bruta } & \multicolumn{2}{c}{ Energia líquida de produção } \\
\cline { 3 - 6 } & Consumo de matéria seca, kg & $\%$ & $\mathrm{~kg}$ & $\mathrm{Mcal} / \mathrm{kg}$ & \multicolumn{1}{c}{ Mcal } \\
\hline Silagem de milho & 9,2 & 8,8 & 0,81 & 1,45 & 13,34 \\
Milho moído & $\mathbf{2 , 6}$ & 9,4 & 0,24 & 2,01 & 5,226 \\
Farelo soja & $\mathbf{3 , 2}$ & 43 & 1,38 & 2,72 & 8,704 \\
Sal branco & 0,8 & - & - & - & - \\
Premix mineral & 0,2 & - & - & - & - \\
\hline Total & 16 & - & 2,43 & - & 27,27 \\
\hline
\end{tabular}

\section{Conclusão}

Antes de iniciar a formulação de uma ração é necessário conhecer os animais que serão alimentados com a dieta e as exigências nutricionais que deverão ser atendidas conforme as características dos animais. Posteriormente serão escolhidos os alimentos que serão utilizados na dieta, e é necessário conhecer suas propriedades físicas e químicas, possibilitando o fornecimento de ingredientes de qualidade e otimizando o uso pelos animais. Por fim, deve-se escolher um método de formulação de ração, este pode ser por softwares com utilização de computadores ou formulações mais simples, que podem ser feitas com o auxílio de uma calculadora. Nunca esquecer, porém, que os animais são seres vivos e, portanto, crescem, adoecem, variam sua produção com o tempo (no caso da produção de leite, por exemplo). Desta forma, sendo o produtor deverá estar ajustando a formulação para melhor atender as reais exigências do momento. Além disso, sempre formulamos para a média de um rebanho, de um lote. Por isso a importância de manter a uniformidade num lote de animais. Do contrário, sempre haverá animais subalimentados e outros superalimentados, o que não é interessante nem para rentabilidade do negócio, nem para a produtividade e persistência da produção.

\section{Referências bibliográficas}

Andriguetto, J. M., Perly, Y., L. \& Minardi, I. (2003). Nutrição Animal (Vol. 66): Editora Nobel.

ANUALPEC. (2019). Anuário da Pecuária Brasileira (20th ed. Vol. 1). São Paulo, São Paulo, Brasil: Instituto FNP. 
Berchielli, T. T., Pires, A. V. \& Oliveira, S. G. (2011). Nutrição de Ruminantes. Jaboticabal, Brazil: FUNEP.

Cappelle, E. R., Valadares Filho, S. C., Silva, J. F. C. \& Cecon, P. R. (2001). Estimativas do valor energético a partir de características químicas e bromatológicas dos alimentos. Revista Brasileira de Zootecnia, 30(6):1837-1856. doi: http://dx.doi.org/10.1590/S1516-35982001000700022.

FAPRI. (2019). Food and Agricultural Policy Research Institute. Food and Agricultural Policy Research Institute, from http://www.fapri.iastate.edu/tools/outlook.aspx

Giger-Reverdin, S., Duvaux-Ponter, C., Sauvant, D., Martin, O., Prado, I. N. \& Müller, R. (2002). Intrinsic buffering capacity of feedstuffs. Animal Feed Science and Technology, 96(1):83-102.

Ibarra, R., Rich, K. M., Adasme, M., Kamp, A., Singer, R. S., Atlagich, M., . . . Escobar-Dodero, J. (2017). Animal production, animal health and food safety: Gaps and challenges in the chilean industry. Food Microbiology1-5.

Lana, R. P. (2007). Sistema Viçosa de formulação de rações (Vol. 1). Viçosa, Minas Gerais: Universidade Federal de Viçosa.

NRC. (1989). Nutrient Requirements of Dairy Cattle (7th rev. ed.). Washington: Natl. Acad. Press, Washington, DC.

NRC. (1994). Nutrients Requirements of Poultry (7th rev. ed.). Washington: Natl. Acad. Press, Washington, DC.

NRC. (2000). Nutrient Requirements of Beef Cattle (7th rev. ed.). Washington, DC, USA: Natl. Acad. Press.

NRC. (2001). Nutrient Requirements of Dairy Cattle (7th rev. ed.). Washington: Natl. Acad. Press, Washington, DC.

NRC. (2007). Nutrient requirements of small ruminants: sheep, goats, cervids, and new world camelids (7th rev. ed.). Washington: Natl. Acad. Press, Washington, DC.

NRC. (2012). Nutrient Requirements of Swine (7th rev. ed.). Washington: Natl. Acad. Press, Washington, DC.

Prado, I. N. (2010). Produção de bovinos de corte e qualidade da carne (Vol. 1). Maringá, Paraná, Brasil: Eduem.

Rezende, P. L. P., Neto, M. D. F., Restle, J., Fernandes, J. J. R., Pádua, J. T. \& Queiroz, G. A. B. (2011). Validação de modelos matemáticos para predição de consumo voluntário e ganho em peso de bovinos. Archivos de Zootecnia, 60(232):921-930.

Rostagno, H. S., Albino, L. F. T., Donzele, J. L., Gomes, P. C., Oliveira, R., Lopes, D. C., . . Euclides, R. F. (2017). Composição de alimentos e exigências nutricionais (3 ed. Vol. 1). Viçosa: Universidade Federal de Viçosa.

Sakomura, N. K. \& Rostagno, H. S. (2007). Métodos de pesquisa em nutrição de monogástricos. Jaboticabal: FUNEP.

Valadares Filho, S. C., Costa e Silva, L. F., Gionbelli, M. P., Rotta, P. P., Marcondes, M. I., Chizzotti, M. L. \& Prados, L. F. (2016). Exigências nutricionais de zebuínos puros e cruzado-BR-Corte (Vol. 1). Viçosa, Minas Gerais: Universidade Federal de Viçosa.

Recebido: 5 de agosto 2019.

Aprovado: 18 de setembro, 2019.

Publicado: 18 de outubro, 2019.

Licenciamento: Este artigo é publicado na modalidade Acesso Aberto sob a licença Creative Commons Atribuição 4.0 (CC-BY 4.0), a qual permite uso irrestrito, distribuição, reprodução em qualquer meio, desde que o autor e a fonte sejam devidamente creditados 